\title{
Study of cord blood lipid profile at birth and its relation to gestational maturity and birth weight
}

\author{
Rashmi Gupta', Alpana Goyal2, Madhuri Gupta ${ }^{3}$ \\ ${ }^{1}$ Associate Professor, ${ }^{2}$ Professor, Department of Biochemistry, SMS Medical College, Jaipur, Rajasthan, India, \\ ${ }^{3}$ Associate Professor, Department of Biochemistry, National Institute of Medical Sciences and Research, Jaipur, \\ Rajasthan, India
}

Background: Dyslipidemia is attributed for coronary artery diseases. Early detection of dyslipidemia in neonatal cord blood could help to identify possible future risk factors of coronary artery diseases in new born. Aims and Objectives: This study was undertaken to compare lipid profile of small for gestational age (SGA) or preterm and appropriate for gestational age (AGA) or near-term neonates. Material and Methods: A total of 60 neonates were included; of which 33 were AGA and 27 SGA neonates. $2 \mathrm{ml}$ of blood was obtained from the umbilical vein immediately after cord clamping at delivery from each subject and kept in a plain vial, after the serum separation analyzed for biochemical parameters. Results: In SGA neonates, mean TC (total cholesterol), TG (triglycerides) LDL (low density lipoprotein cholesterol), HDL (high density lipoprotein cholesterol) and triglycerides were $66.60 \pm 17.76 \mathrm{mg} / \mathrm{dl}, 57.32 \pm 23.7 \mathrm{mg} / \mathrm{dl}, 54.06 \pm 20.11 \mathrm{mg} / \mathrm{dl}, 19.06 \pm 17.03 \mathrm{mg} / \mathrm{dl}$ respectively. SGA neonates had more TC, TG and LDL than AGA neonates $(P<0.05)$. There was significant difference in mean lipid profile between preterm andnear-term neonates. Thus, lipid profiles in SGA neonates in thisstudy are higher than that of AGAneonates. Conclusion: It can be concluded that preterm babies are exposed to more hypercholesterolemia and potentially more atherogenic environment than their term counterparts.

Key words: Cord blood Lipid profile; Small gestational age (SGA); Appropriate gestational age (AGA); Newborn

\section{INTRODUCTION}

Worldwide the most common cause of disability and death in adults is cardiovascular disease (CVD). Moreover, several maternal and fetal factors Such as hypertension, diabetes, obesity, and low or high birth weight, can influence fetal blood lipid levels. ${ }^{1}$ The fetus needs a considerable amount of cholesterol for the development of tissues and organs. After birth, human lipid transportation system changes from containing low levels of very-low-density lipoprotein (VLDL), and low-density lipoprotein (LDL), to the adult pattern, this continues to increase with age. ${ }^{2}$
LDL is the major cholesterol-carrying particle in the plasma. HDL is responsible for transporting cholesterol back from the tissues to the liver. Race and gender differences in lipoproteins levels have repeatedly been demonstrated in adults. ${ }^{3}$ The cord blood cholesterol level in infants is lower than the adults. ${ }^{4}$ Small for gestational age (SGA) Infants has higher levels of triglyceride, rich VLDL, and intermediate low-density lipoprotein (LDL), in comparison with the AGA (appropriate for gestational age) infants. These findings suggest a link between higher triglyceride, rich VLDL subclasses in SGA infants, and future coronary artery disease. ${ }^{5}$ 
These differences have also been noted in children, supporting the concept that the variance is due to genetic influences, rather than environmental factors.Observations from both epidemiological and clinical studies have suggested that the pathological process of coronary artery disease begins in childhood. The aortas of children as young as 3-4 years age often contain initial lipid deposits, commonly called "fatty streaks". Various studies have shown an enhanced rate of coronary heart disease among men and women whose birth weights were at the lower end of the normal range and who were thin or short at birth. ${ }^{6,7}$ Low birth weight is associated with increased incidence of cardiovascular disease, hypertension and type 2 diabetes. ${ }^{8}$ The aim of this study was to estimate the lipid profile excluding apolipoproteins in neonates and look for an association with birth weight and gestational age.

\section{MATERIAL AND METHODS}

\section{Study design and location}

This observational study was conducted for a period of six months in the department of Biochemistry in association with department of gynecology and obstetrics, SMS Medical College and hospital, Jaipur on 60 newborns. All newborns were having gestational age in between 24-42 weeks without any congenital abnormalities with vaginal delivery. The study was approved by the institutional ethical committee and all the parents of the neonates gave written informed consent for the study.

A thorough clinical examination of the new born was done and weight of the baby was calculated by electronic weighing scale. Classification of infants was done on gestational age as term and preterm new born based on New Ballard's scoring.

\section{Inclusion criteria}

The new-borns were divided into 3 categories based on birth weight as those with a birth weight of 1) $<2.5 \mathrm{Kg}, 2$ ) between $2.5-3.5 \mathrm{~kg}$ and 3)> $3.5 \mathrm{~kg}$ based on of gestational age. The new born were also divided into 2 sub-groups based on gestational maturity- 1) Preterm 34 weeks of gestational age 2) Near-term $=35-37$ weeks of gestational age.

\section{Exclusion criteria}

Neonates with Congenital malformations, family history of coronary heart disease/ hypercholesterolemia, with prenatal problems like hypoglycaemia, pathological jaundice and maternal illness like Diabetes mellitus (DM) including Insulin dependent diabetes mellitus (IDDM) and gestational diabetes, Tuberculosis, Asthma, Pregnancy induced hypertension, Drug abuse in mother and antenatal medications and neonates with Instrumental delivery including extraction were excluded from this study.

\section{Sampling procedure}

The gestational age was determined according to the date of the last menstrual period, or the early ultrasound in 20 weeks of gestation. Blood samples were selected for analysis in a random order. All the information related to the newborns and their mothers were recorded in the prescribed format. 2 $\mathrm{ml}$ of blood was obtained from the umbilical vein immediately after cord clamping at delivery from each subject and kept in a plain vial. Serum was separated by centrifugation at $3000 \mathrm{rpm}$ for 10 minutes and taken in another test tube for biochemical analysis on fully automatic analyzer (Randox RX Imola). Total cholesterol, triglycerides and HDL were measured using an enzymatic method. Serum LDL was estimated using Friedewald's Formula (LDLCholesterol $=$ Total cholesterol (HDL cholesterol + triglycerides / 5 \{VLDL-C $\}$ ).

\section{Statistical analysis}

All data were represented as mean $\pm S D$. Mean values of variables were compared using unpaired students t- test. Data was compiled and analyzed by using software SPSS 16.0. Ap value $<0.05$ was considered statistically significant.

\section{RESULTS}

Out of 60 new-borns 33(55\%) were preterm babies and $27(45 \%)$ were near-term. Table 1 shows the comparison of demographic characteristics and lipid profile between two subgroups of neonates. Mean values of serum triglycerides, total cholesterol and LDL levels were high in preterm neonates,but the difference was statistically significant only with serum triglycerides and LDL values $(p<0.05)$. Mean value of HDL was high in near-term neonates compared to preterm neonates. However, the difference was not statistically significant with serum HDL values ( $p>0.05)$.

Table 2 shows the comparison of lipid profile parameters and Chol/HDL ratio between normal birth weight and low birth weight neonates. Mean values for serum TC, TG and LDL were found to be increased with decreasing birth weight while HDL mean values were increased with increasing birth weight,thus by unpaired student's t- test the difference between all lipid profile values were statistically significant.

\section{DISCUSSION}

Numerous studies have been conducted comparing the cord blood lipid profile in preterm and near-term neonates. This study shows that gestational age and birth weight in preterm neonates was lower than those in nearterm neonates. This difference is found to be statistically 


\begin{tabular}{|c|c|c|c|}
\hline Parameters & $\begin{array}{c}(S G A) \text { or } \\
\text { Preterm } \\
(n=33)\end{array}$ & $\begin{array}{c}(A G A) \text { or } \\
\text { Near-term } \\
(n=27)\end{array}$ & $P$ value \\
\hline Age (wk.) & $31.48 \pm 1.43$ & $34.77 \pm 1.88$ & $<0.05^{\star}$ \\
\hline Weight $(\mathrm{kg})$ & $2.04 \pm 1.23$ & $2.75 \pm 1.36$ & $<0.05^{*}$ \\
\hline Total Cholesterol(mg/dl) & $66.60 \pm 17.76$ & $61.0 \pm 14.14$ & 0.18 \\
\hline Triglyceride $(\mathrm{mg} / \mathrm{dl})$ & $67.32 \pm 23.07$ & $54.62 \pm 22.67$ & $<0.05^{\star}$ \\
\hline $\mathrm{LDL}(\mathrm{mg} / \mathrm{dl})$ & $39.32 \pm 16.65$ & $30.22 \pm 5.93$ & $<0.05^{*}$ \\
\hline $\mathrm{HDL}(\mathrm{mg} / \mathrm{dl})$ & $19.06 \pm 17.03$ & $20.74 \pm 19.12$ & 0.7204 \\
\hline
\end{tabular}

*P value $=\leq 0.05$ is significant, (SGA) Preterm $=\leq 34$ weeks of gestational age, (AGA) near-term $=35-37$ weeks of gestational age

\begin{tabular}{|c|c|c|c|}
\hline Lipids & $\begin{array}{c}\text { Normal birth } \\
\text { weight } \\
(2.5-2.9 \mathrm{Kg}) \\
n=25\end{array}$ & $\begin{array}{c}\text { Low birth } \\
\text { weight } \\
(\leq 2.4 \mathrm{Kg}) \\
n=35\end{array}$ & $P$ value \\
\hline Total Cholesterol(mg/dl) & $62.56 \pm 22.3$ & $68.61 \pm 26.6$ & $<0.05^{*}$ \\
\hline Triglyceride (mg/dl) & $49.13 \pm 18.76$ & $57.16 \pm 23.4$ & $<0.05^{\star}$ \\
\hline $\operatorname{LDL}(\mathrm{mg} / \mathrm{dl})$ & $48.8 \pm 15.3$ & $51.77 \pm 19.2$ & $<0.05^{*}$ \\
\hline HDL (mg/dl) & $27.9 \pm 14.9$ & $24.5 \pm 11.2$ & $<0.05^{\star}$ \\
\hline Cholestrol/ HDL & $2.09 \pm 1.01$ & $2.93 \pm 1.89$ & $<0.05^{*}$ \\
\hline
\end{tabular}

significant and it showed that TG and TC level are higher in low birth weight new born.

From this study, it was evident that the mean value of serum TC was higher in preterm than near-term neonates. It has been reported that the serum depletion of cholesterol that occurs at near-term is due to a decrease in LDL-C levels. LDL-C serum levels are low in near-term neonates, most likely due to its rapid uptake and metabolism by the fetal adrenal gland as precursor or substrate for steroid hormone biosynthesis. ${ }^{1}$ Nermin Ramy et al also found significantly increased levels of TGL and LDL in theSGA(small for gestational age) group compared with the AGA (appropriate for gestational age) group. ${ }^{9}$

On comparing the cord blood lipid profile of preterm and near-term HDL - C, in preterm neonates was lower than those of near-term neonates because of LCAT activity (Lecithin Cholesterol Acyl Transferase, also called phosphatidylcholine-sterolO-acyltransferase is an enzyme that convert free cholesterol into cholesteryl ester) was lower in preterm neonates than the term neonates and so higher HDL-C was noted in near-term in comparing with preterm. ${ }^{10-12}$

In this study triglyceride level was also higher in preterm neonates as compared to near-term neonates. The difference was statistically significant. The major role of triglyceride is store energy for later use, so in the preterm energy can be required for the survival of fetus so it can be said that in the preterm TG level was higher than nearterm.In preterm, increase in cord blood cholesterol level may reflect the metabolic adaptation to provide adequate energy, especially to vital organs like brain. ${ }^{13}$

It is found that decrease of lipoprotein lipase play a critical role in breaking down fat in the form of triglycerides, which are carried from various organs to the blood by molecules, hepatic lipase (play a vital role in TG hydrolase and ligand /bridging factor for receptor-mediated lipoprotein uptake), and lecithin cholesterol acyltransferase (LCAT) enzymes activity in near-term newborns, might increase lipoprotein concentrations in Low birth weight than in normal birth weight infants. These enzymes increase the level of lipid profile in LBW. ${ }^{14}$ Contrasting results were obtained in a study done by Asadollah Kermani et al with low TG levels in preterm neonates with an unexplained reason. ${ }^{15}$

The findings in our study thus reaffirm the link between prenatal factors and cord blood lipid profile. There is a significantly unhealthy lipid profile among the preterm babies when compared to near-terms. Thus, we can safely say that preterm's are exposed to more hypercholesterolemic and potentially more atherogenic environment than their term counterparts.

\section{CONCLUSION}

Estimation of serum lipoproteins during birth and early childhood could be predictive for lipoprotein disorders and CVD since low birth weight is an important risk factor for CVD, especially in low income countries. ${ }^{16}$ Our study has suggested that high cholesterol and Triglycerides levels are more likely to be observed in pre-termand low birth weight infants So it seems early prevention and close follow-up would help to prevent the complications of atherosclerosis in these patients and would decrease the disease burden.

\section{Limitation}

The cross-sectional nature of this study is its major limitation. Future longitudinal studies with long term follow up will be required to correlate the current findings with clinical implications.

\section{ACKNOWLEDGEMENT}

The authors are deeply indebted to all the participants of the study. 


\section{REFERENCES}

1. Parker Jr CR, Carr BR, Simpson ER, MacDonald PC. Decline in the concentration of low-density lipoprotein cholesterol in human fetal serum near-term. Metabolism.1983; 919-923. https://doi.org/10.1016/0026-0495(83)90207-X

2. Kharb S, Kaur R., Singh V and Sangwan K. Birth weight, cord blood lipoprotein and apolipoprotein levels in Indian newborns. Int J Prev Med. 2010;1(1):29-33.

3. Loughrey CM, Rimm E, Heiss G and Rifai N. Race and gender differences in cord blood lipoproteins. Atherosclerosis. 2000; 148(1):57-65.

https://doi.org/10.1016/S0021-9150(99)00238-5

4. Bansal N, Cruickshank JK, McElduff P and Durrington PN. Cord blood lipoprotein and prenatal influences. Current Opinion in Lipidology. 2005;16(4):400-408.

https://doi.org/10.1097/01.mol.0000174154.61307.16

5. Kwitervich PO, Cockrill SL, Virgil DG, Garrett ES, Otvos J, Driggers $\mathrm{R}$, et al. Lipoprotein heterogeneity at birth: influence of gestational age and race on lipoprotein subclasses. Ethn Dis Summer. 2004; 14(3):351-359.

6. Barker DJP, Osmond C, Winter PD, Margetts B and Simmonds SJ. Weight in infancy and death from ischaemic heart disease. Lancet. 1989; 334:577-580.

https://doi.org/10.1016/S0140-6736(89)90710-1

7. Holme I. An analysis of randomized trials evaluating the effect of cholesterol reduction on total mortality and coronary heart disease incidence. Circulation. 1990; 82(6): 1916-1924.

https://doi.org/10.1161/01.CIR.82.6.1916

8. Zanetti D, Tikkanen E, Gustafsson S, James R. Priest, Burgess S and Ingelsson E. Birthweight, Type 2 Diabetes Mellitus, and Cardiovascular Disease: Addressing the Barker Hypothesis with Mendelian Randomization. Circulation: Genomic and Precision Medicine. 2018;11(6):1-9.
https://doi.org/10.1161/CIRCGEN.117.002054

9. Nermin Ramy, Zakaria M, El Kafoury M and Kamal M. Cord blood lipid profile in relation to anthropometric measures of newborns . Kasr Al Ainy Medical Journal. 2017; 23:54-58. https://doi.org/10.4103/kamj.kamj_5_17

10. Pardo IMCG, Geloneze B, Tambascia MA and Barros- Filho AA. Atherogenic Lipid Profile of Brazilian Near-Term Newborns. Braz J Med Biol Res. 2005; 38(5):755-760. https://doi.org/10.1590/S0100-879X2005000500013

11. Avinash NJ, Arun K. Tadas and Swati AT. Lipid profile of umbilical cord blood of near-term and preterm neonates. International Journal of Current Medical and Applied Sciences 2014; PP:01-11

12. Atiy JK, Manther AA and Nadhim MS. Lipid Profile in Cord Blood and its Relation to Selected Neonatal and Maternal Characteristics. J Neonatal Biol. 2018; 7: 269. doi:10.4172/21670897.1000269

13. Kenchappa $Y$ and Behera N. Assay of neonatal cord blood lipid levels and its correlation with neonatal gestational age, gender and birth weight: a single center experience. Int J Contemp Pediatr. 2016; 3:71 https://doi.org/10.18203/2349-3291.ijcp20161905

14. Kelishadi R, Haghjooy J S, Tajadini M and Mansourian M. Genetic association with low concentrations of high-density lipoprotein cholesterol in a paediatric population of middle east and north Africa. Atherosclerosis. 2014; 237(1):273-278. https://doi.org/10.1016/j.atherosclerosis.2014.08.043

15. Kermani A, Namakin K, Sharifzadeh GR and Faal GR. Umbilical Cord Blood Lipid Profile in Healthy Neonates in Iran. Iran J Pediatr. 2020; 30 (3): e99114. https://doi.org/10.5812/ijp.99114

16. Kelishadi R, Derakhshan R, Sabet B, Sarraf-Zadegan N, Kahbazi M, Sadri $\mathrm{GH}$, et al. The metabolic syndrome in hypertensive and normotensive subjects: The Isfahan Healthy Heart Programme. Ann Acad Med Singapore. 2005; 34:243-249.

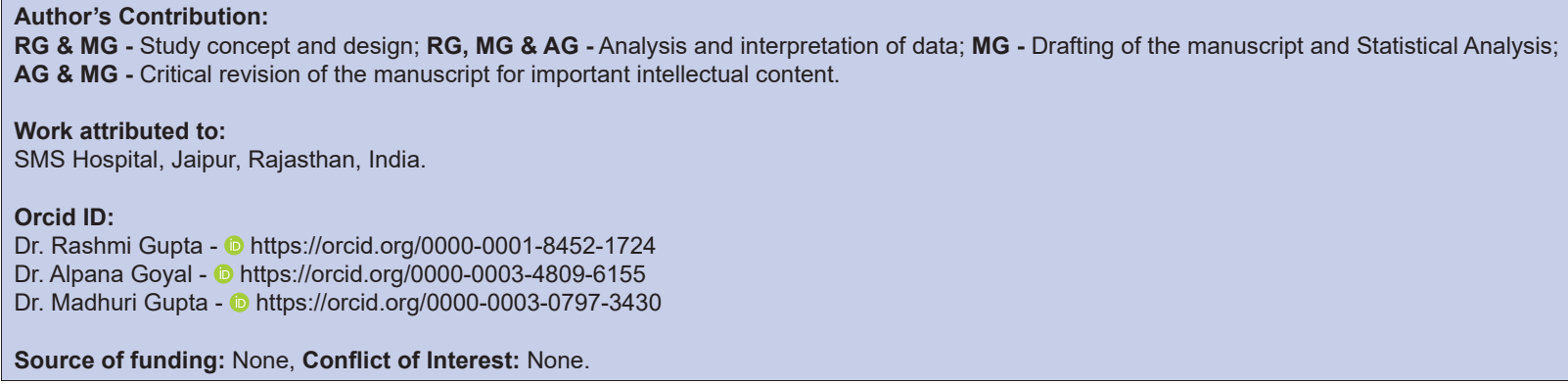

